\title{
Extended haematological follow-up after parenteral artesunate in African children with severe malaria
}

\author{
Thierry Rolling ${ }^{1,2^{*}}$, Dorothee Spahlinger ${ }^{1}$, Saadou Issifou ${ }^{3}$, Tsiri Agbeniyega ${ }^{4}$, Peter G Kremsner ${ }^{3,5}$ \\ Gerd D Burchard ${ }^{1,2}$, Benjamin Mordmueller ${ }^{3,5}$, Jakob P Cramer ${ }^{1,2}$ \\ From Challenges in malaria research \\ Basel, Switzerland. 10-12 October 2012
}

\section{Background}

Mortality of severe malaria is significantly lower in patients treated with parenteral artesunate when compared to quinine. No significant side effects have been described from previous studies but follow-up periods have been limited.

First reports from Europe have shown cases of posttreatment haemolysis occurring two weeks after the first dose of parenteral artesunate. Furthermore, haemolysis was accompanied by a delay in adequate reticulocytopoiesis for up to two weeks. All patients developing posttreatment haemolysis had hyperparasitaemia. The aim of this study was to gain information about the incidence, clinical impact and pathophysiological background of this effect in the hyperendemic setting of sub-Saharan Africa.

\section{Materials and methods}

This study was conducted as a substudy to the ongoing Severe Malaria in African Children Follow-up study and was implemented in Lambaréné, Gabon and Kumasi, Ghana. 50 patients were recruited per study site.

Patients were randomized to different treatment regimes of parenteral artesunate ( 3 doses of $4 \mathrm{mg} / \mathrm{kg}$ iv, im or 5 doses of $2.4 \mathrm{mg} / \mathrm{kg}$ im respectively). Haematological parameters and serum markers of haemolysis and erythropoiesis (lactate dehydrogenase, haptoglobin, bilirubine, soluble transferrin receptor and erythropoietin) were determined on days $0,7,14$ and 28 . The clinical impact of changes in haemoglobin was assessed.

'Department of Internal Medicine, Section of Tropical Medicine, University

Medical Centre Hamburg-Eppendorf, Hamburg, Germany

Full list of author information is available at the end of the article

\section{Results and conclusions}

The preliminary results show a delay in the reticulopoietic response after treatment of severe malaria, which is accompanied by a slower recovery of haemoglobin levels in hyperparasitaemic patients $(>100,000$ parasites $/ \mu \mathrm{l})$, when compared to low parasitaemic patients. Hyperparasitaemic patients had a mean rise of $1.1 \mathrm{~g} / \mathrm{dL}$ in $\mathrm{Hb}$ between days 0 and 28, while patients with low parasitaemia had a mean rise of $2.1 \mathrm{~g} / \mathrm{dL}$ between days 0 and 28 . First results on the potential pathophysiologic background including haemolysis and $I$ or impaired erythropoiesis will be presented. In conclusion, there seems to an association between parasite levels and recovery of haemoglobin levels. Whether or not this effect is associated with malaria disease, treatment with parenteral artesunate or both is subject of further investigations.

\section{Author details \\ ${ }^{1}$ Department of Internal Medicine, Section of Tropical Medicine, University Medical Centre Hamburg-Eppendorf, Hamburg, Germany. ${ }^{2}$ Department of Clinical Research, Bernhard-Nocht-Institute for Tropical Medicine, Hamburg, Germany. ${ }^{3}$ Medical Research Unit, Albert Schweitzer Hospital, Lambaréné, Gabon. ${ }^{4}$ Kwame Nkrumah University of Science and Technology School of Medicine, Kumasi, Ghana. ${ }^{5}$ Institute of Tropical Medicine, University of Tübingen, Germany.}

Published: 15 October 2012

doi:10.1186/1475-2875-11-S1-P83

Cite this article as: Rolling et al:: Extended haematological follow-up after parenteral artesunate in African children with severe malaria. Malaria Journal 2012 11(Suppl 1):P83. 